\title{
Fatores de risco para osteólise do enxerto do coracoide após o procedimento de Latarjet aberto
}

\section{Risk Factors for Coracoid Graft Osteolysis after the Open Latarjet Procedure}

\author{
Marcio Cohen ${ }^{10} \quad$ Alexandre Dreifus Zaluski ${ }^{1} \quad$ Glaucio Sales de Lima Siqueira ${ }^{1}$ \\ Marcus Vinicius Galvão Amaral ${ }^{1}$ Martim Teixeira Monteiro ${ }^{1}$ Geraldo Rocha Motta Filho ${ }^{1}$ \\ ${ }^{1}$ Departamento Cirurgia do Ombro e Cotovelo, Instituto Nacional de \\ Traumatologia e Ortopedia, Ministério da Saúde ,Rio de Janeiro, \\ RJ, Brasil \\ Rev Bras Ortop 2020;55(5):585-590. \\ Endereço para correspondência Marcio Cohen, MD, Departamento \\ Cirurgia do Ombro e Cotovelo, Instituto Nacional de Traumatologia e \\ Ortopedia, Ministério da Saúde (INTO-MS), Rua Almirante Guilhem, \\ 127, Leblon, Rio de Janeiro, 22440-000, Brasil \\ (e-mail: cohenmarcio@gmail.com).
}

\section{Resumo}

\section{Palavras-chave}

- instabilidade articular

- luxação do ombro

- articulação do ombro

- reabsorção óssea

- osteólise
Objetivo Osteólise do processo coracoide é descrita como uma possível complicação da cirurgia de Latarjet. O objetivo do presente estudo foi avaliar a incidência e fatores de risco associados à osteólise do enxerto do coracoide e correlacioná-los com resultados clínicos.

Métodos Foi realizada uma revisão retrospectiva incluindo 38 casos submetidos ao procedimento de Latarjet. Em todos os casos, foi realizada uma tomografia computadorizada antes e pelo menos 1 ano após a cirurgia. A presença de osteólise do coracoide foi avaliada e correlacionada com os seguintes fatores de risco: idade, tabagismo, e perda óssea pré-operatória da glenóide. Os pacientes foram divididos em dois grupos: A (ausência ou menor reabsorção óssea) e B (maior reabsorção óssea ou total). A avaliação funcional foi determinada através do escore de Rowe.

Resultado Osteólise do processo coracoide ocorreu em 22 casos (57,8\%). O defeito ósseo médio pré-operatório da glenóide foi de $22,8 \%$ no grupo $A$ e de $13,4 \%$ no grupo $B$ $(p=0.0075)$. A média de idade dos casos em ambos os grupos não apresentou diferença estatística. Tabagismo também não esteve relacionado com diferenças no resultado. Não houve correlação entre a presença de osteólise e o arco de movimento, dor ou ao escore de Rowe. Não houve casos de reluxação; entretanto, quatro pacientes apresentaram apreensão anterior no exame físico.

Conclusão A reabsorção do processo coracoide ocorreu em pelo menos $50 \%$ dos pacientes submetidos à cirurgia de Latarjet, e a ausência pré-operatória de perda óssea significativa da glenóide foi o único fator de risco associado a osteólise mais severa do enxerto, porém sem influência no resultado clínico.

Objective Coracoid osteolysis has been described as a possible complication after the Latarjet procedure. The aim of the present study was to investigate the incidence and risk factors associated to coracoid graft osteolysis and to correlate them with clinical results. recebido

03 de Junho de 2019

aceito

23 de Julho de 2019
DOI https://doi.org/

10.1055/s-0039-1698799. ISSN 0102-3616.
Copyright $\odot 2020$ by Sociedade Brasileira License terms de Ortopedia e Traumatologia. Published by Thieme Revinter Publicações Ltda, Rio de Janeiro, Brazil 


\author{
Keywords \\ - joint instability \\ - shoulder \\ dislocation \\ - shoulder joint \\ - bone resorption \\ - osteolysis
}

Methods A retrospective review of 38 Latarjet procedures was conducted. Computed tomography (CT) scans were obtained from all of the patients before and at least 1 year after the surgery. Coracoid osteolysis was evaluated and correlated to preoperative factors, namely: age, smoking status, and preoperative glenoid bone loss. The patients were divided into 2 groups: A (no or minor bone resorption) and B (major or total bone resorption). The functional outcome was determined by the Rowe score.

Results Coracoid graft osteolysis occurred in 22 cases (57.8\%). The mean preoperative glenoid defect was $22.8 \%$ in group $A$, and $13.4 \%$ in group $B(p=0.0075)$. The mean ages of the subjects in both groups were not significantly different. Smoking did not seem to affect the main outcome either, and no correlation was found between graft osteolysis and postoperative range of motion, pain, or Rowe score. There were no cases of recurrent dislocations in our sample, although four patients presented with a positive anterior apprehension sign.

Conclusion Bone resorption of the coracoid graft is present in at least $50 \%$ of the patients submitted to the Latarjet procedure, and the absence of significant preoperative glenoid bone loss showed to be the only risk factor associated with severe graft osteolysis, even though this did not influence significantly the clinical outcome.

\section{Introdução}

Nos últimos anos, muitos artigos foram publicados sobre os resultados do procedimento de bloqueio ósseo descrito por Latarjet para o tratamento da instabilidade glenoumeral anterior. ${ }^{1-4}$ Mesmo com algumas modificações de cirurgião para cirurgião, o princípio básico da cirurgia continua a ser a transferência do coracoide através do tendão subescapular. ${ }^{1,5-7}$ Esse procedimento se tornou o tratamento de escolha em casos com deficiências ósseas > 20\% da área da superfície glenóide, na cirurgia de revisão, e em atletas de esportes de contato de alto risco, mesmo no contexto de deficiência óssea limitada. ${ }^{8}$ Para obtenção dos melhores resultados e menos complicações, o posicionamento adequado do enxerto é essencial para que a consolidação entre o processo do coracoide e a glenóide seja nivelada. ${ }^{9-12}$ A reabsorção do enxerto ósseo coracoide pode ocorrer mesmo após a consolidação óssea. Esse fenômeno tem sido relatado, e alguns autores consideram que ele pode estar associado a dor no ombro e à recidiva da luxação. ${ }^{3,4,11,13}$ Di Giacomo et al. ${ }^{14}$ foram uns dos primeiros autores a relatar a localização e a quantidade de osteólise do enxerto de coracoide após o procedimento de Latarjet usando análise de tomografia computadorizada (TC). Ele também publicou outro estudo que afirma que pacientes com perda óssea da glenóide pré-operatória (> 15\%) sofreram menos osteólise em comparação àqueles com defeitos menores. ${ }^{15} \mathrm{Di}$ Giacomo acredita que, em pacientes sem perda óssea da glenóide, não há estímulos mecânicos da cabeça do úmero para certas áreas do enxerto, o que pode contribuir para a reabsorção do coracoide. O objetivo do presente estudo foi avaliar a incidência de osteólise do enxerto ósseo após o procedimento de Latarjet e correlacioná-la à idade, tabagismo e presença de perda óssea pré-operatória da glenóide. Nossa hipótese foi que, como descrito anteriormente, a osteólise do enxerto ósseo é mais pronunciada nos casos sem perda óssea da glenóide.

\section{Materiais e Métodos}

\section{População do Estudo}

Este é um estudo observacional retrospectivo de 72 pacientes submetidos à cirurgia para tratamento de instabilidade anterior do ombro por meio do procedimento de Latarjet entre maio de 2012 e agosto de 2016. Os critérios de inclusão foram disponibilidade de realização de TC pré-operatória e pelo menos 1 ano após a cirurgia e de exame físico pelo menos 2 anos após o procedimento. Casos com grande desvio da técnica mais comumente utilizada (como a técnica de arco congruente), pacientes submetidos a retirada do material de osteossintese ou aqueles sem pronta consolidação do enxerto foram excluídos. Todos os pacientes consentiram por escrito em participar do estudo, que foi aprovado pelo comitê de ética do instituto.

O principal desfecho estudado foi a osteólise do enxerto coracoide, classificado em quatro tipos segundo Zhu et al. ${ }^{16}$ (-Tabela 1). De acordo com essa classificação, os pacientes foram divididos em dois grupos, A (sem reabsorcão ou menor) e B (reabsorção maior ou total). Os grupos foram

Tabela 1 Classificação de Zhu et al. ${ }^{16}$ da reabsorção pósoperatória de osso coracoide (quatro graus, com base em imagem de tomografia computadorizada axial). A reabsorção óssea foi classificada como grau mais elevado entre os dois parafusos

Grau 0 (sem reabsorção): O cone da cabeça do parafuso é enterrado no enxerto ósseo coracoide

Grau I (reabsorção menor): Somente a cabeça do parafuso é exposta fora do enxerto ósseo.

Grau II (reabsorção maior): Parte da haste do parafuso é exposta fora do enxerto.

Grau III (reabsorção total): A cabeça do parafuso e a haste estão totalmente expostas, todo o enxerto ósseo coracoide foi absorvido e não há osso no colo da glenóide. 
comparados em várias exposições, como a quantidade de perda óssea da glenóide antes da cirurgia, tabagismo, o uso de âncoras de sutura, e idade. Também buscamos a correlação entre a reabsorção do enxerto e os resultados clínicos pós-operatórios usando o escore de Rowe, a escala analógica visual e a amplitude de movimento. As variáveis foram obtidas na TC realizada antes e pelo menos 1 ano após a cirurgia. A amplitude de movimento foi avaliada no exame físico, e a dor foi registrada pela escala analógica visual. Estes resultados foram inseridos em uma planilha do Microsoft Excel (Microsoft Corporation, Redmond, WA, EUA), e a análise estatística foi feita com o programa Real Statistics (Charles Zaionitz).

\section{Técnica Cirúrgica}

A cirurgia foi realizada sob anestesia geral combinada com bloqueio interescalênico. Os pacientes foram colocados em posição de cadeira de praia. Uma incisão cirúrgica foi feita a partir do processo coracoide e se estendeu por $\sim 7 \mathrm{~cm} \mathrm{em}$ sentido inferior. Através do intervalo deltopeitoral, o processo coracoide foi osteotomizado com um osteótomo curvo. O tendão subescapular foi dividido horizontalmente ao longo da fibra muscular à altura do terço inferior da largura do tendão. Uma incisão vertical foi feita na cápsula articular anterior. Tanto o enxerto coracoide quanto o colo da glenóide foram submetidos à remoção do córtex antes da fixação com 2 parafusos (parafusos corticais de $3,5 \mathrm{~mm}$ ou esponjosos de $4,0 \mathrm{~mm}$ ) sem qualquer guia específico. De acordo com a preferência dos cirurgiões, a cápsula anterior era recolocada na borda inferior anterior da glenóide nativa com uma âncora de sutura bioabsorvível Gryphon (Depuy Synthes, Warsaw, IN, EUA) ou suturada no coto do ligamento coracoacromial ou não recolocada. Uma tipoia protegeu o ombro por 4 semanas após a cirurgia. A amplitude passiva de movimento foi iniciada em 2 semanas, e a atividade diária foi permitida em 4 semanas. 0 fortalecimento do ombro foi iniciado 3 meses após a cirurgia.

\section{Imagem Radiográfica}

As imagens foram obtidas em tomógrafo de 64 canais Philips Brilliance (Philips, Amsterdã, Holanda), a 250 mA, 120 Kvp e espessura de corte de $1 \mathrm{~mm}$ ). A perda óssea pré-operatória da glenóide foi medida em uma reconstrução tridimensional (3D) da face glenoidal com subtração da cabeça do úmero. A porcentagem de perda óssea da glenóide foi medida pelo método de área de superfície do programa Image J (National Institutes of Health, Bethesda, MA, EUA) com conceito de "círculo de melhor ajuste". ${ }^{17-20}$ A TC pós-operatória foi feita depois de, no mínimo, 1 ano. A consolidacão, posição e reabsorção do enxerto foram avaliadas. A consolidacão foi confirmada pela ponte com osso entre o bloco ósseo e a glenóide. A presença de uma linha radiotransparente completa entre o enxerto e a glenóide representou a não união e o caso foi excluído. Se o córtex lateral do coracoide era $>1 \mathrm{~mm}$ medial ou lateral à superfície articular da glenóide, a posição do coracoide era definida como saliente medial ou lateral, respectivamente. Caso contrário, se a cortical lateral do coracoide estivesse a $1 \mathrm{~mm}$ da superfície da glenóide, a posição do coracoide era definida como nivelada (flush). 0 sistema de classificação descrito por Zhu et al. ${ }^{16}$ foi utilizado para avaliar a reabsorção do enxerto coracoide (-Tabela $\mathbf{1}$ ).

\section{Avaliação Estatística}

Os resultados foram registrados em uma planilha de Excel, e a análise estatística foi feita em Real Statistics. Os pacientes foram divididos nos dois grupos, e a normalidade foi avaliada pelo teste de Shapiro-Wilk. Variáveis contínuas com distribuição normal foram comparadas pelo teste $t$ de Student (defeitos pré-operatórios da glenóide). As variáveis de distribuição não normal foram comparadas pelo teste de MannWhitney (idade e escore de Rowe). Dados categóricos com amostras pequenas foram comparados pelo teste direto de Fischer (uso de âncoras de sutura e tabagismo).

\section{Resultados}

Dos 72 pacientes inicialmente incluídos no estudo, 13 foram excluídos por falta de exames pré-operatórios, e 11 por falta de exames pós-operatórios. Três pacientes foram excluídos por se submeterem à remoção do implante durante a cirurgia de revisão no $1^{\circ}$ ano. Dois apresentaram não união do enxerto e, em seis, o coracoide foi colocado com a superfície inferior côncava voltada para a articulação (técnica de arco congruente). Depois das exclusões, permaneceram 38 ombros de 37 pacientes. A média de idade foi de 33 anos ( 21 a 67 anos); 32 pacientes eram do sexo masculino, e 5 do sexo feminino. O tamanho médio do defeito pré-operatório da glenóide foi de 20\% (0 a 34\%). Um total de 27 casos apresentou lesão óssea de Bankart, e 10 casos apresentaram erosão óssea. Um caso não tinha defeito. Em 11 casos, a cápsula anterior foi recolocada na glenóide com uma âncora. Em nove casos, não houve utilização de âncora e a cápsula foi suturada ao coto do ligamento coracoacromial ou à cabeça do parafuso (sete e dois casos, respectivamente). Nos últimos 18 casos, a reinserção da cápsula não foi realizada. A avaliação pós-operatória por TC foi realizada em um tempo médio de acompanhamento de 27 meses ( 12 a 72 meses). A posição do enxerto foi considerada ótima em 34 casos. Três casos foram considerados mediais, e um, lateral. Segundo o sistema de classificação de Yi-Ming Zhu et al., ${ }^{16}$ a reabsorção do enxerto ocorreu em 22 casos (57,8\%), sendo 11 do tipo I, 8 do tipo II, e 3 do tipo III. Em todos os casos, a reabsorção mais grave se deu na parte superior do enxerto, ao redor do parafuso superior (-Fig.1). Assim, havia 27 casos no grupo A (graus 0 e I) e 11 casos no grupo B (graus II e III). O defeito médio préoperatório da glenóide foi de $22,8 \%$ no grupo A e de $13,4 \%$ no grupo B $(p=0,0075)$ (-Tabela 2$)$. Não houve diferença significativa na média de idade dos indivíduos dos dois grupos: 32 e 35,8 anos nos grupos A e B, respectivamente. Não foi encontrada correlação entre a osteólise do enxerto e o uso da âncora de sutura para reparo capsular (6 de 27 versus 5 de 11), amplitude de movimento pós-operatória, dor ou escore de Rowe ( 88,3 versus 89$)(p=0,46)$. O tabagismo não parece afetar o resultado principal, embora houvesse apenas dois indivíduos fumantes em cada grupo. Não houve casos de instabilidade recorrente em nossa amostra, embora quatro 

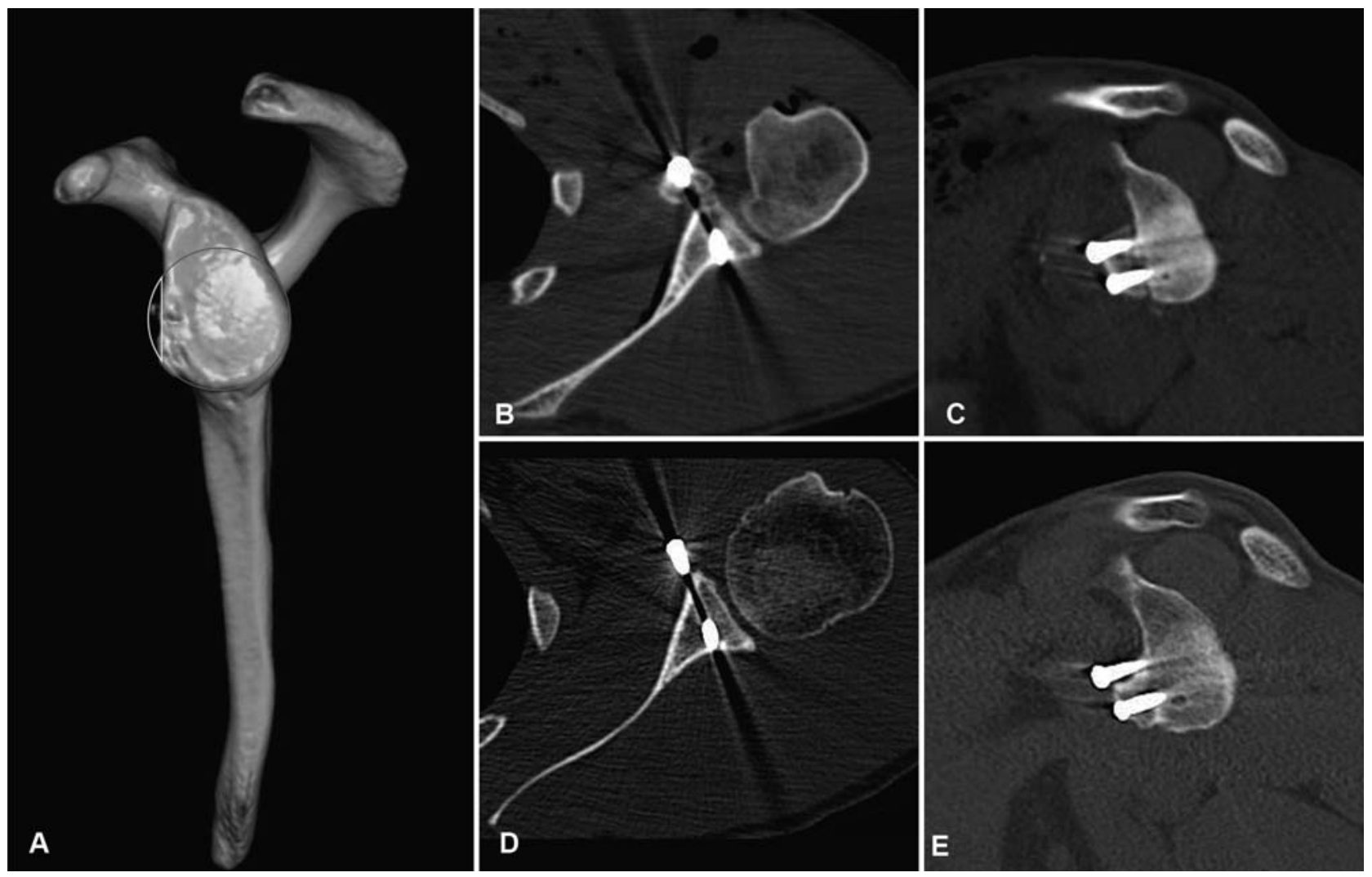

Fig. 1 (A) Tomografia computadorizada pré-operatória de reconstrução tridimensional da glenóide. A borda anterior do defeito da glenóide (em amarelo) e a circunferência do círculo perfeito (em vermelho) foram delineadas. Porcentagem de perda óssea =(área superficial do defeito/área superficial do círculo perfeito da glenóide) $\times 100 \%$. (B, C) Tomografia axial e sagital no pós-operatório imediato. (D, E) A tomografia computadorizada realizada 42 meses após a cirurgia mostra a reabsorção grau III da parte superior do enxerto coracoide.

Tabela 2 Características dos Grupos A e B

\begin{tabular}{|l|l|l|l|}
\hline & $\begin{array}{l}\text { Grupo A } \\
\text { (grau 0/I) }\end{array}$ & $\begin{array}{l}\text { Grupo B } \\
\text { (grau II/III) }\end{array}$ & valor-p \\
\hline Casos & 27 & 11 & \\
\hline $\begin{array}{l}\text { Acompanhamento } \\
\text { (meses) }\end{array}$ & $\begin{array}{l}27,9 \\
(12-67)\end{array}$ & $\begin{array}{l}26,9 \\
(12-72)\end{array}$ & \\
\hline $\begin{array}{l}\text { Perda óssea } \\
\text { pré-operatória da } \\
\text { glenóide (área) }\end{array}$ & $\begin{array}{l}22,8 \% \\
(7-34)\end{array}$ & $\begin{array}{l}13,4 \% \\
(0-24)\end{array}$ & 0,0075 \\
\hline Idade (anos) & 32 & 35,8 & 0,410 \\
\hline Tabagismo & 2 & 2 & 0,562 \\
\hline Âncora & 6 & 4 & 0,424 \\
\hline
\end{tabular}

pacientes, todos do grupo A, apresentassem teste positivo de apreensão anterior (-Tabela $\mathbf{3}$ ).

O enxerto coracoide estava bem posicionado e não apresentava reabsorção nos quatro casos com apreensão anterior positiva.

\section{Discussão}

O procedimento de Latarjet é um método confiável para prevenir a instabilidade recorrente anterior do ombro, e muitos autores relatam resultados bem-sucedidos. ${ }^{21-24}$ Ao mesmo tempo, poucos estudos mencionam a condição da
Tabela 3 Comparação dos resultados clínicos entre os grupos A e B

\begin{tabular}{|l|l|l|l|}
\hline & $\begin{array}{l}\text { Grupo A } \\
\text { (grau 0/I) }\end{array}$ & $\begin{array}{l}\text { Grupo B } \\
\text { (grau II/III) }\end{array}$ & valor-p \\
\hline Escore de Rowe & $88(45-100)$ & $89(65-100)$ & 0,46 \\
\hline $\begin{array}{l}\text { Elevação para } \\
\text { a frente }\end{array}$ & $\begin{array}{l}160^{\circ} \\
(130-180)\end{array}$ & $\begin{array}{l}151^{\circ} \\
(140-160)\end{array}$ & 0,175 \\
\hline Rotação externa & $51^{\circ}(30-80)$ & $48^{\circ}(25-70)$ & 0,585 \\
\hline Rotação interna & T9 (T6-L1) & T10 (T5-L3) & 0,349 \\
\hline $\begin{array}{l}\text { Apreensão } \\
\text { anterior }\end{array}$ & 4 & 0 & 0,309 \\
\hline Recidiva & 0 & 0 & \\
\hline
\end{tabular}

reabsorção óssea coracoide. O presente estudo mostra que há alguma reabsorção óssea do enxerto coracoide em pelo menos 50\% dos pacientes submetidos à cirurgia de Latarjet, e que pacientes com menor perda óssea pré-operatória da glenóide foram mais propensos a apresentar osteólise mais grave do enxerto. Uma das teorias relacionadas a esse achado é que há falta de estímulos mecânicos do enxerto em pacientes com menor perda óssea da glenóide, enquanto pacientes com defeitos mais significativos estariam sujeitos a forças de manutenção quando o coracoide substitui o grande defeito ósseo (lei de Wolff). ${ }^{14,15}$ Assim, constatamos que a parte superior do enxerto apresentava reabsorção mais 
pronunciada em todos os pacientes. Acreditamos que isso ocorra porque a parte inferior do coracoide é submetida a forças de tração do tendão conjunto, sendo protegida da reabsorção. Podemos também especular que esta parte tenha alguma contribuição de vascularização do tendão conjunto. Di Giacomo et al. ${ }^{14}$ fizeram um estudo para determinar a localização e a quantidade de osteólise do enxerto do coracoide após o procedimento de Latarjet usando TC. Esses autores descreveram uma classificação dividindo o coracoide em oito partes e identificaram a reabsorção mais pronunciada na parte superficial e medial. Foi a primeira classificação publicada sobre este assunto, e os autores acreditavam que os resultados da reabsorção se deviam a fatores mecânicos e biológicos. ${ }^{14}$ Posteriormente, os mesmos autores publicaram um segundo trabalho correlacionando a presença de perda óssea da glenóide e osteólise do enxerto coracoide e identificaram menor osteólise em pacientes com perda óssea significativa da glenóide $(>15 \%){ }^{15}$

Zhu et al. ${ }^{16}$ descreveram um sistema de classificação mais simples do que Giacomo para avaliar a gravidade da reabsorção óssea do coracoide transferido. Sua classificação é baseada na quantidade de reabsorção observada na TC axial em torno de cada um dos parafusos; a reabsorção é classificada pelo grau mais elevado entre os dois parafusos. Em nosso estudo, o maior grau foi sempre no parafuso superior. Esses autores observaram incidência de 90,5\% de reabsorção óssea coracoide após 1 ano e, no nosso estudo, utilizando a mesma classificação, encontramos uma incidência de $57,8 \%$. Zhu et al. ${ }^{16}$ não tentaram correlacionar a incidência ou a gravidade da reabsorção com a perda óssea da glenóide, e todos os pacientes de sua série tinham um defeito ósseo pré-operatório $>20 \%$ na glenóide. Haeni et al. ${ }^{25}$ realizaram um estudo prospectivo com 15 pacientes após o procedimento de Latarjet artroscópico e verificaram que a metade superior do coracoide sofre uma significativa quantidade de osteólise após 6 meses. Uma diferença de seu estudo foi que a reabsorção foi avaliada através de tomografias tridimensionais com análise volumétrica. Não houve menção de implicações clínicas ou de complicações devido à reabsorção. Recentemente, Zhu et al. ${ }^{26}$ publicaram um estudo comparativo prospectivo entre o Latarjet aberto e artroscópico e observaram menor reabsorção do enxerto no grupo artroscópico. Ambos os grupos não apresentaram diferença significativa quanto à perda óssea pré-operatória da glenóide. Os autores atribuíram esse resultado a uma causa multifatorial de reabsorção e acreditavam que a melhor proteção dos tecidos moles durante a cirurgia artroscópica poderia aumentar a preservação do suprimento sanguíneo do coracoide. Uma das principais preocupações sobre a osteólise do processo coracoide é a possível associação a piores resultados clínicos, mas, até o momento, não há consenso. ${ }^{4,25}$ Um total de 11 pacientes (29\%) da nossa série teve reabsorção maior (graus II e III), mas isso não foi correlacionado a qualquer diferença nos desfechos funcionais ou ao risco de recidiva da instabilidade em comparação a pacientes sem reabsorção ou com reabsorção menor. Lunn et al., ${ }^{27}$ em uma série de pacientes nos quais o procedimento de Latarjet foi ineficaz, identificaram a lise do enxerto como um fator de risco para recorrência da instabilidade. Isso pode gerar uma discussão sobre a importância do bloqueio ósseo em comparação com ao efeito tirante do tendão conjunto na estabilidade após o procedimento de Latarjet. $^{28,29}$ Nossos resultados clínicos demonstraram que a presença de reabsorção não estava relacionada à maior incidência de recidiva. Di Giacommo et al. ${ }^{14,15}$ sugerem que o efeito do bloqueio ósseo pode não ser o principal devido à enorme osteólise observada em seus pacientes. Poderíamos especular que a contribuição óssea para a estabilidade após o procedimento de Latarjet é mais importante em casos com defeitos ósseos maiores na glenóide porque esses são os pacientes com menos osteólise, como já demonstrado.

O número limitado de pacientes e o curto período de acompanhamento são algumas das limitações do presente estudo. Embora não tenhamos identificado um efeito negativo no desfecho clínico dos pacientes com reabsorção mais grave do enxerto, ainda existem preocupações sobre este assunto. Portanto, recomendamos cautela ao realizar os procedimentos de Latarjet em casos sem perda óssea significativa da glenóide, considerando a maior chance de reabsorção da parte superior do enxerto; além disso, a posição ideal do parafuso deve ser assegurada.

\section{Conclusão}

O presente estudo mostra que reabsorção óssea do enxerto coracoide está presente em pelo menos $50 \%$ dos pacientes submetidos à cirurgia de Latarjet, e que a ausência de defeito significativo pré-operatório da glenóide parece ser o único fator de risco associado à osteólise mais grave do enxerto, apesar da ausência de importância clínica significativa nesses casos.

\section{Observação}

Comitê de Revisão Institucional (IRB) - Plataforma Brasil, número CAAE 68193617.8.0000.5273.

Trabalho desenvolvido no Departamento de Cirurgia do Ombro e Cotovelo, Instituto Nacional de Traumatologia e Ortopedia, Ministério da Saúde (INTO-MS), Rio de Janeiro, RJ, Brasil.

\section{Conflitos de Interesses}

Os autores declaram que não há conflito de interesses.

\section{Referências}

1 Boileau P, Thélu CÉ, Mercier N, et al. Arthroscopic Bristow-Latarjet combined with bankart repair restores shoulder stability in patients with glenoid bone loss. Clin Orthop Relat Res 2014;472 (08):2413-2424

2 da Silva LA, da Costa Lima AG, Kautsky RM, et al. Evaluation of the results and complications of the Latarjet procedure for recurrent anterior dislocation of the shoulder. Rev Bras Ortop 2015;50(06): 652-659

3 Hovelius L, Sandström B, Sundgren K, et al. One hundred eighteen Bristow-Latarjet repairs for recurrent anterior dislocation of the shoulder prospectively followed for fifteen years: study I-clinical results. J Shoulder Elbow Surg 2004;13(05):509-516 
4 Mizuno N, Denard PJ, Raiss P, et al. Long-term results of the Latarjet procedure for anterior instability of the shoulder. J Shoulder Elbow Surg 2014;23(11):1691-1699

5 Hovelius L, Körner L, Lundberg B, et al. The coracoid transfer for recurrent dislocation of the shoulder. Technical aspects of the Bristow-Latarjet procedure. J Bone Joint Surg Am 1983;65(07): 926-934

6 Ikemoto RY, Murachovisky J, Nascimento LG, et al. Results from Latarjet surgery for treating traumatic anterior shoulder instability associated with bone erosion in the glenoid cavity, after minimum follow-up of one year. Rev Bras Ortop 2015;46(05):553-560

7 Latarjet M. [Treatment of recurrent dislocation of the shoulder]. Lyon Chir 1954;49(08):994-997

8 Shaha JS, Cook JB, Song DJ, et al. Redefi “critical” bone loss in shoulder instability. Am J Sports Med 2015;43(07):1719-1725

9 Athwal GS, Meislin R, Getz C, et al. Short-term complications of the arthroscopic Latarjet procedure: a North American experience. Arthroscopy 2016;32(10):1965-1970

10 Casabianca L, Gerometta A, Massein A, et al. Graft position and fusion rate following arthroscopic Latarjet. Knee Surg Sports Traumatol Arthrosc 2016;24(02):507-512

11 Samim M, Small KM, Higgins LD. Coracoid graft union: a quantitative assessment by computed tomography in primary and revision Latarjet procedure. J Shoulder Elbow Surg 2018;27 (08):1475-1482

12 Gartsman GM, Waggenspack WN Jr, O'Connor DP, et al. Immediate and early complications of the open Latarjet procedure: a retrospective review of a large consecutive case series. J Shoulder Elbow Surg 2017;26(01):68-72

13 Balestro JC, Young A, Maccioni C, et al. Graft osteolysis and recurrent instability after the Latarjet procedure performed with bioabsorbable screw fixation. J Shoulder Elbow Surg 2015; 24(05):711-718

14 Di Giacomo G, Costantini A, de Gasperis N, et al. Coracoid graft osteolysis after the Latarjet procedure for anteroinferior shoulder instability: a computed tomography scan study of twenty-six patients. J Shoulder Elbow Surg 2011;20(06):989-995

15 Di Giacomo G, de Gasperis N, Costantini A, et al. Does the presence of glenoid bone loss influence coracoid bone graft osteolysis after the Latarjet procedure? A computed tomography scan study in 2 groups of patients with and without glenoid bone loss. J Shoulder Elbow Surg 2014;23(04):514-518

16 Zhu YM, Jiang CY, Lu Y, et al. Coracoid bone graft resorption after Latarjet procedure is underestimated: a new classification system and a clinical review with computed tomography evaluation. J Shoulder Elbow Surg 2015;24(11):1782-1788

17 Hamamoto JT, Leroux T, Chahla J, et al. Assessment and Evaluation of Glenoid Bone Loss. Arthrosc Tech 2016;5(04):e947-e951

18 Huysmans PE, Haen PS, Kidd M, et al. The shape of the inferior part of the glenoid: a cadaveric study. J Shoulder Elbow Surg 2006;15 (06):759-763

19 Magarelli N, Milano G, Sergio P, et al. Intra-observer and interobserver reliability of the 'Pico' computed tomography method for quantification of glenoid bone defect in anterior shoulder instability. Skeletal Radiol 2009;38(11):1071-1075

20 Sugaya H, Moriishi J, Dohi M, et al. Glenoid rim morphology in recurrent anterior glenohumeral instability. J Bone Joint Surg Am 2003;85(05):878-884

21 Allain J, Goutallier D, Glorion C. Long-term results of the Latarjet procedure for the treatment of anterior instability of the shoulder. J Bone Joint Surg Am 1998;80(06):841-852

22 Gordins V, Hovelius L, Sandström B, et al. Risk of arthropathy after the Bristow-Latarjet repair: a radiologic and clinical thirty-three to thirty-five years of follow-up of thirty-one shoulders. J Shoulder Elbow Surg 2015;24(05):691-699

23 Lafosse L, Boyle S. Arthroscopic Latarjet procedure. J Shoulder Elbow Surg 2010;19(2, Suppl)2-12

24 Rosa JRP, Checchia CS, Miyazaki AN. Traumatic anterior instability of the shoulder. Rev Bras Ortop 2017;52(05):513-520

25 Haeni DL, Opsomer G, Sood A, et al. Société Française d'Arthroscopie. Three-dimensional volume measurement of coracoid graft osteolysis after arthroscopic Latarjet procedure. J Shoulder Elbow Surg 2017;26(03):484-489

26 Zhu Y, Jiang C, Song G. Arthroscopic Versus Open Latarjet in the Treatment of Recurrent Anterior Shoulder Dislocation With Marked Glenoid Bone Loss: A Prospective Comparative Study. Am J Sports Med 2017;45(07):1645-1653

27 Lunn JV, Castellano-Rosa J, Walch G. Recurrent anterior dislocation after the Latarjet procedure: outcome after revision using a modified Eden-Hybinette operation. J Shoulder Elbow Surg 2008; 17(05):744-750

28 Dines JS, Dodson CC, McGarry MH, et al. Contribution of osseous and muscular stabilizing effects with the Latarjet procedure for anterior instability without glenoid bone loss. J Shoulder Elbow Surg 2013;22(12):1689-1694

29 Yamamoto N, Itoi E, Abe $\mathrm{H}$, et al. Effect of an anterior glenoid defect on anterior shoulder stability: a cadaveric study. Am J Sports Med 2009;37(05):949-954 